\title{
Nonequilibrium Measures Which Exhibit a Temperature Gradient: Study of a Model
}

\author{
A. Galves ${ }^{1 \star}$, C. Kipnis ${ }^{2 \star \star}$, C. Marchioro $^{3}$, and E. Presutti ${ }^{4}$ \\ 1 Instituto de Matématica e Estatistica, Universidade de São Paulo, São Paulo, Brazil \\ 2 Centre de Mathématiques de l'Ecole Polytechnique, Plateau de Palaiseau, F-91128 Palaiseau Cedex, \\ France \\ 3 Dipartimento di Matematica, Libera Università di Trento, I-38050 Povo (Trento), Italy \\ 4 Istituto Matematico, Università di Roma, I-00100 Roma, Italy
}

\begin{abstract}
We give some rules to define measures which could describe heat flow in homogeneous crystals. We then study a particular model which is explicitly solvable: the one dimensional nearest neighborhood Ising model. We analyze two cases. In the first one the spins at the two boundaries interact with reservoirs at different temperatures; in the thermodynamical limit the measure we introduce converges locally to Gibbs measures and a temperature profile is so derived. We obtain an explicit expression for the thermal conductivity coefficient which depends on the temperature. In the second case we study the asymptotic behavior starting from an initial state in which each half of the space is at a different temperature. We find again a temperature profile which asymptotically obeys the heat equation with the thermal conductivity coefficient previously derived. From a mathematical point of view, the analysis of the invariant measure is made possible by studying a "time-reversed" process related to a graphical representation of an associated process. This provides us with an explicit formula for the $n$-fold correlation function and we study the limiting behavior using both this representation (for proving an exchangeability result) and a Donsker-type, spacetime renormalization procedure.
\end{abstract}

\section{Introduction}

In this paper we study the stationary nonequilibrium measures which describe systems where a temperature gradient is present and a heat flow is established. Even though this is a classical problem in Statistical Mechanics, many questions still remain unanswered, particularly in the framework of a mathematically rigorous approach. The main point of investigation $[1,2]$ concerns both a general characterization of these measures and the (dynamical) way the stationary heat flow is established. In this paper we restrict ourselves to the first aspect so as to

* Partially supported by CNPq grant No. 402876/79

$\star \star$ Laboratoire de Recherche Associé au CNRS No. 169 
avoid the deep difficulties connected with the second. The price we pay is high: without dynamical considerations the definition we give of the state describing heat flow is quite arbitrary, it becomes in fact the hypothesis of the theory rather than being the result of it. To support our assumptions we can only give a few motivations and some heuristic considerations; we would therefore rather look at its consequences. At the moment we can exhibit a (quite explicit) solution of a particular model. Possible further studies in this direction are briefly discussed in the last section.

We begin by recalling some physical questions which arose quite naturally in [2] and that give some insight on the motivations which inspired our hypotheses. In [2] a system of point particles is investigated. The particles move in a bounded region $\Lambda$ and interact pairwise. When they collide with $\partial \Lambda$ their velocities are stochastically reflected with a Maxwellian law whose parameter depends on where in $\partial \Lambda$ the collision takes place and corresponds to the temperature of a reservoir in thermal contact with the system. Under suitable hypotheses on the interaction it is proven that the state of the system approaches a unique (invariant) measure. However the state so obtained does not necessarily exhibit a heat flow; this depends on the nature of the interaction among particles which should satisfy some hypotheses of dissipativity. For instance, even if the particles were not interacting, uniqueness would hold, but in this case Fourier's law would not be satisfied. The same conclusion holds in Spohn and Lebowitz, [1], for a chain of harmonic oscillators. We introduce below a "local ergodic assumption" (for lattice systems where mass transport phenomena are absent, see the Concluding Remarks) which may more appropriately be called a "local equilibrium condition" since dynamics does not enter in our approach. ${ }^{1}$ We will then investigate the way these local conditions "match" together to build a measure for the whole system.

Usually equilibrium states at temperature $T$ (DLR measures) are introduced by requiring that any subsystem be in a local equilibrium defined by the Gibbs measure at temperature $T$. In order to describe systems in thermal contact with reservoirs at different temperatures we therefore assume that the equilibrium "at the boundaries" is described by Gibbs distributions at the temperatures of the reservoirs. For the "internal subsystems" local equilibrium should be defined differently since there is no common temperature throughout the system.

We say that a subsystem is in local equilibrium if all configurations with the same energy have the same weight. This implies that the state is Gibbs if all subsystems of a given size not too small are in local equilibrium and if the temperatures at the boundaries are the same. Also for infinite systems, where the boundary conditions in some sense disappear, local equilibrium, as above, implies that the state is DLR, more precisely a superposition of DLR states, because of micro canonical-DLR equivalence. These statements hold for a quite general class of interactions, see for instance [3] and references quoted therein.

1 It is indeed possible to prove approach to the local equilibrium, as we define it, for some quantum mechanical system coupled to suitable reservoirs (infinite systems of particles) in the so-called "weak coupling limit". This is considered in [13] where in particular (in the above limit) it is shown that a system of finitely many quantum spins goes to a stationary measure, which is just the one we will be studying in this paper. We are indebted to A. Frigerio for pointing out this reference 
It can be seen that generally no measure exists which has at the same time all the above subsystems in local equilibrium, if the temperature at the boundaries is not uniform, i.e. there is no solution for these "equilibrium equations", no probability measure has conditional probabilities given by our requirements of local equilibrium. We are therefore led to weaken again our demands, and we proceed as follows.

We introduce a Markov process by randomly choosing, each time, a subsystem (of a given fixed size) and then defining a transition probability which leads that subsystem to its "local equilibrium": uniform distribution on its energy surface for internal subsystems, Gibbs distribution at the reservoirs' temperatures for those at the boundaries. We assume that the measure which describes the heat flow in the system is the measure which is invariant under the above transition probability, i.e. stationary under the corresponding "stochastic dynamics".

In this paper we study the consequences of the above assumption in a particular model which is somehow explicitly solvable: the nearest neighbourhood one dimensional Ising model. We consider spins $S_{x}\left[S_{x}= \pm 1\right]$ at sites $-L, \ldots, L$ and we randomly choose $x$ in a uniform way. While the assumption of uniformity is somehow natural for internal $x$ 's, because of the homogeneity of the space, one could think that different rates of choice could be given to $x$ in the neighbourhood of the boundaries. We will see that this does not lead to any change in the thermodynamical limit, $L$ going to infinity (Sect. 4, concluding remarks).

If $-L+1 \leqq x \leqq L-1$ then the transition probability changes $S_{y}$ only for $y=x$ and in such a way that the energy

$$
U\left(S_{x} \mid S_{x+1}, S_{x-1}\right)=-J S_{x-1} S_{x}-J S_{x} S_{x+1}
$$

is unchanged. $S_{x}$ is then distributed uniformly on $U=$ const. If $x=L[-L]$ then the transition probability changes only $S_{L}\left[S_{-L}\right]$ and it.is given by

$$
\frac{e^{\beta_{2} J S_{L}^{\prime} S_{L-1}}}{e^{\beta_{2} J}+e^{-\beta_{2} J}}
$$

and analogously for $x=-L$, with $\beta_{1} \neq \beta_{2}, \beta=\frac{1}{k T}$.

It is easy to show (see Sect. 2) that for any $L>0$ there is only one measure invariant under the above stochastic dynamics. We are interested in its asymptotic behavior as $L \rightarrow \infty$ and we want to show that it exhibits a temperature gradient. To make this apparent we proceed as follows.

Let $\mathcal{O}$ be the algebra of local observables [4] (cylindrical bounded functions) then for any $\xi \in(-1,+1)$ we define the "measure at $\xi L " v_{\xi}^{(L)}$ as

$$
v_{\xi}^{(L)}(f)=\mu^{(L)}\left(\tau_{[\xi L]} f\right), \quad f \in \mathcal{O},
$$

where $\mu^{(L)}$ is the stationary measure for the region $[-L, L], \tau, y \in \mathbb{Z}$, is the translation by $y,[\xi L]$ is the integer part of $\xi L$. The measure $v_{\xi}^{(L)}$ expresses "the structure of the state around $\xi L "$.

We use this definition to exploit the variation of $v_{\xi}^{(L)}$ versus $\xi$, as $L$ goes to infinity. We prove that for each $\xi, v_{\xi}^{(L)}$ approaches a DLR measure at temperature $T(\xi)$. We prove that $T(\xi)$ is a smooth monotonic function between $T_{1}$ and $T_{2}$, the 
boundary temperatures. From this, if we assume the validity of Fourier's law, we obtain the thermal conductivity coefficient $k(T)$. More precisely let $Q\left(T_{1}, T_{2}\right)$ be the heat flux. Fourier's law is

$$
Q\left(T_{1}, T_{2}\right)=-K(T) \frac{d T}{d \xi}
$$

and so we obtain the ratio $K(T) / K\left(T_{0}\right)$ in terms of the profile $T(\xi), T$ and $T_{0}$ are temperatures between $T_{1}$ and $T_{2}$. It turns out that in our model $K(T) / K\left(T_{0}\right)$ is well defined, namely it does not depend on $T_{1}$ and $T_{2} . K(T)$ is found to be, up to a multiplicative factor, the specific heat (for the Ising model). On the other hand we can avoid assuming Fourier's law and in fact we can prove it for our model, if we use in a stronger way the stochastic evolution so that it defines not only the stationary measure but also the heat flux $Q\left(T_{1}, T_{2}\right)$. The argument reads as follows : $Q_{L}\left(T_{1}, T_{2}\right)$, the heat flux at finite volume, $[-L, L]$, is just the (total) energy change per unit time due to switches of the spin at $L$; the energy change due to the spin at $-L$ is just the opposite and represents the heat per unit time transferred to the reservoir at temperature $T_{1}$. If we adjust the time scale in the stochastic evolution so that it converges to an "infinite volume" dynamics, see Sect. 2, we find out that $Q_{L}\left(T_{1}, T_{2}\right)$ goes to zero as $1 / L$ and we define $Q\left(T_{1}, T_{2}\right)$ as $\lim _{L \rightarrow \infty} L Q_{L}\left(T_{1}, T_{2}\right)$. Furthermore, up to this order, $Q_{L}$ is proportional to $-\frac{1}{L} \frac{d T}{d \xi}$. This proves Fourier's law. Furthermore the ratio $-L Q_{L}\left(T_{1}, T_{2}\right) / \frac{d T}{d \xi}$ defines the same heat conductivity coefficient as before.

The stochastic dynamics we introduced allows us to study also another model for the heat flow. We consider an initial state $\mu_{0}$ described by Gibbs distributions at different temperatures $T_{2}, T_{1}$ in the right and left half spaces. If we denote by $\mu_{t}$ the distribution at time $t$, we find that asymptotically in $t, \mu_{t}$ locally looks like a Gibbs measure with space-time dependent temperature $T(i, t), i \in \mathbb{Z}$. This can be "naturally" approximated by a smooth function $T(x, t), x \in \mathbb{R}$. If we consider the heat equation (for heat conductivity coefficient depending on the temperature) we find the following: the function $T(x, t)$ is a solution of this equation provided that the specific heat $C$ is that of the Ising model, and $K$, the heat conductivity coefficient, is the one previously derived (by suitably fixing the multiplicative coefficient).

From a technical point of view we derive an "explicit" expression for the $n$-fold correlation functions of the invariant measure via a sort of time reversed process. To perform this we build a particular graphical representation of our original motion using the ideas of "association" [8]. The reversed process reduces our computation to a system of simple random walks with exclusion and absorption at the boundaries. We identify the limit of the correlation functions by using first a Donsker-type renormalization which is shown to converge to independent Brownian motions for the two-site correlation, and an exchangeability argument to get the full statement. Both these limiting procedures rely on classical estimates of the number of returns to the origin for simple random walks on $\mathbb{Z}$.

In Sect. 2 we give the main results, in Sect. 3 the probability estimates needed for the proofs of Sect. 2 and in Sect. 4 some concluding remarks. 


\section{Results}

We consider the region $-L, \ldots, L, L \in \mathbb{N}$, and for $x \in[-L, L]$ spins $S_{x}= \pm 1$. The interaction energy (nearest neighborhood Ising model) is

$$
\begin{aligned}
U\left(S_{x} \mid S_{x+1}\right) & =-J S_{x} S_{x+1} \\
U\left(S_{x} \mid S_{x-1}, S_{x+1}\right) & =-J S_{x-1} S_{x}-J S_{x} S_{x+1} .
\end{aligned}
$$

The transition probability $P\left(S, S^{\prime}\right), S, S^{\prime} \in\{-1,1\}^{2 L+1}\left(S, S^{\prime}\right.$ are spins configuration in $[-L, L])$ is defined to be

$$
\begin{aligned}
& P\left(S, S^{\prime}\right)= \sum_{x=-L+1}^{L-1} \frac{1}{2 L+1} \frac{\delta\left[U\left(S_{x}^{\prime} \mid S_{x-1}, S_{x+1}\right)-U\left(S_{x} \mid S_{x-1}, S_{x+1}\right)\right]}{\#\left(U\left(S_{x} \mid S_{x-1}, S_{x+1}\right)\right)} \prod_{y \neq x} \delta\left(S_{y}-S_{y}^{\prime}\right) \\
&+\frac{1}{2 L+1} \frac{e^{+\beta_{2} J S_{L}^{\prime} S_{L-1}}}{e^{-\beta_{2} J}+e^{\beta_{2} J}} \prod_{y<L} \delta\left(S_{y}-S_{y}^{\prime}\right)+\frac{1}{2 L+1} \frac{e^{+\beta_{1} J S^{\prime}-L S}-L+1}{e^{-\beta_{1} J}+e^{\beta_{1} J}} \\
& \cdot \prod_{y>-L} \delta\left(S_{y}-S_{y}^{\prime}\right), \\
& \delta(a)=\left\{\begin{array}{lll}
1 & \text { if } & a=0 \\
0 & \text { if } & a \neq 0
\end{array}\right. \\
& \quad \#\left(U\left(S_{x} \mid S_{x-1}, S_{x+1}\right)\right)=\operatorname{Card}\left\{S_{x}^{\prime}: U\left(S_{x}^{\prime} \mid S_{x-1}, S_{x+1}\right)=U\left(S_{x} \mid S_{x-1}, S_{x+1}\right)\right\} .(2.2 \mathrm{~b})
\end{aligned}
$$

In Eq. (2.2) the terms with $x \in[-L+1, L-1]$ express the achievement of a microcanonical local equilibrium while at $x=-L[+L]$ the equilibrium is Gibbs at temperature $\beta_{1}\left[\beta_{2}\right]$. Notice that in the semi-infinite DLR distribution [6] the probability that the first spin is $S_{L}^{\prime}$, given the boundary condition $S_{L-1}$, is written as

$$
\frac{e^{+\beta_{2} J S_{L}^{\prime} S_{L-1}}}{e^{-\beta_{2} J}+e^{\beta_{2} J}}
$$

which is just the term accurring in Eq. (2.2).

Proposition 2.1. There exists a unique probability measure $\mu_{L}(S), S \in\{-1,+1\}^{2 L+1}$ which is invariant under $P$, i.e.

$$
\sum_{S} \mu_{L}(S) P\left(S, S^{\prime}\right)=\mu_{L}\left(S^{\prime}\right)
$$

Proof. It is easy to see that $P$ is an irreducible, aperiodic Markov chain. From a classical theorem on Markov chains the thesis follows, [5].

As discussed in the introduction, the limit of $\mu_{L}$ should be studied in the topology defined by the following:

Definition 2.1. Let $\mathcal{O}$ be the algebra of local (cylindrical) bounded functions on $\{-1,+1\}^{\mathbb{Z}}$. Namely if $S \in\{-1,+1)^{\mathbb{Z}}, f(S) \in \mathcal{O}$ if $f$ is bounded and if there is $M>0$ such that $f$ only depends on $S_{-M}, \ldots, S_{M}$. Let

$$
\begin{gathered}
\forall a, x \in \mathbb{Z}:\left(\hat{\tau}_{a} S\right)_{x}=(S)_{x+a}, \\
\left(\tau_{a} f\right)(S)=f\left(\hat{\tau}_{a} S\right), \quad f \in \mathcal{O} .
\end{gathered}
$$


We consider $\mu_{L}$, defined in Proposition 2.1, as a probability measure on the whole space $\{-1,+1\}^{\mathbb{Z}}$ by saying for instance that with probability $1: S_{x}=1 \forall x:|x|>L$. Then we define $v_{\xi}^{(L)}$ on $\{-1,+1\}^{\mathbb{Z}}$ as

$$
\begin{aligned}
v_{\xi}^{(L)}(f)= & \mu_{L}\left(\tau_{[\xi L]} f\right), \quad \forall f \in \mathcal{O} \\
& \xi \in(-1,+1) \\
{[\xi L]=} & \text { integer part of } \xi L .
\end{aligned}
$$

[By Kolmogorov's theorem, Eq. (2.6) uniquely defines $v_{\xi}^{(L)}$.]

Theorem 2.1. For every $\xi \in(-1,+1), v_{\xi}^{(L)}(f)$ converges for any $f \in \mathcal{O}$ (when $L$ diverges). The limit defines a probability measure $v_{\xi}$ which is DLR at a temperature $T(\xi)$ (implicitly) defined by

$$
\begin{array}{r}
f(\beta(\xi))=f\left(\beta_{1}\right) \frac{1-\xi}{2}+f\left(\beta_{2}\right) \frac{1+\xi}{2}, \\
f(\beta)=\frac{e^{\beta J}-e^{-\beta J}}{e^{\beta J}+e^{-\beta J}}, \quad \beta=\frac{1}{K T} .
\end{array}
$$

$\beta_{1}, \beta_{2}$ are defined by Eq. (2.2).

Proof. The proof is given in the next section.

Theorem 2.1 determines the heat conductivity coefficient $K(T)$ if we assume that Fourier's law holds in our case : let $Q\left(T_{1}, T_{2}\right)$ be the heat flux; Fourier's law is

$$
Q\left(T_{1}, T_{2}\right)=-K(T) \frac{d}{d \xi} T(\xi) .
$$

Then from equation (2.7)

$$
K(T)=\text { const. } \frac{d}{d T} f(\beta(T)), \quad \beta(T)=\frac{1}{K T},
$$

where the const. in Eq. (2.9) is $K\left(T_{0}\right)\left[\left.\frac{d}{d T} f(\beta(T))\right|_{T=T_{0}}\right]^{-1}$ for some fixed $T_{0} . K(T)$ is then proportional to the specific heat $[f(\beta)$ is in terms proportional to the mean energy of the system]. As explained in the introduction, the important feature in Eq. (2.9) is that the dependence on $\beta_{1}$ and $\beta_{2}$ has disappeared so that $K(T)$ has been defined consistently. In fact $K(T)$ does not change even if we somehow vary either the interaction with the reservoir or the size of the subsystem which is assumed to go to local equilibrium (see Sect.4). To obtain the explicit formula Eq. (2.9) for $K(T)$ we do not really need to assume Fourier's law. This can in fact be proven to hold (in our model) provided that we use in a stronger way the stochastic evolution so as to define not only the stationary measure but also the heat flux $Q\left(T_{1}, T_{2}\right)$. The argument follows:

The heat transferred to the system from the reservoirs in an interval of time is measured by the corresponding change of total energy of the system (use the first law of thermodynamics, noticing that in our model no "work" is done on the 
system). Since the change in energy is due to switches of the spins at $-L$ or at $+L$ we can say from where, left or right, the heat has been transferred to. Therefore we pose the

Definition 2.2. Consider $L$ as fixed. Let $(S(n))_{n \in \mathbb{N}} \in\left(\{-1,+1\}^{2 L+1}\right)^{\mathbb{N}}$ so that

$$
S(n)=\left\{S_{-L}(n), \ldots, S_{L}(n)\right\}
$$

is a spin configuration and $(S(n))_{n \in \mathbb{N}}$ a possible trajectory for the stochastic evolution. The heat (per unit time) given to the system at time $n+1$ from the right reservoir is the random variable

$$
\delta Q_{L}(n)=J\left[\left|S_{L}(n+1)-S_{L-1}(n+1)\right|-\left|S_{L}(n)-S_{L-1}(n)\right|\right] \delta\left(S_{L-1}(n+1)-S_{L-1}(n)\right) .
$$

The heat from the left reservoir $\delta Q_{-L}(n)$ is defined similarly. A heat flux can also be defined in a natural way inside $[-L, L]$. Let $x=-L-1, \ldots, L-1, E_{x}$ the energy of the spins $-L, \ldots, x$. Then

$$
\delta Q_{x}(n)=J\left[\left|S_{x}(n+1)-S_{x-1}(n+1)\right|-\left|S_{x}(n)-S_{x-1}(n)\right|\right] \delta\left(S_{x-1}(n+1)-S_{x-1}(n)\right)
$$

is the heat flux at $x$ at time $n+1$ coming from the left. The average flux per unit time is defined by

$$
\delta Q_{j}=\lim _{N \rightarrow \infty} \frac{1}{N} \sum_{n=0}^{N-1} \delta Q_{j}(n), \quad j=-L, \ldots, L,
$$

which is well posed because of

Proposition 2.2. The limit in Eq. (2.11) exists for all $j$ and a.s. with respect to the (stationary) process $P_{\mu}^{(L)}$, with initial (invariant) measure $\mu_{L}$. The $\delta Q_{j}$ are a.s. constant and $\delta Q_{j}=-\delta Q_{L}$ for $j=-L, \ldots, L-1$ :

$$
\delta Q_{-L}=-\delta Q_{L}=\frac{2 J}{4 L(2 L+1)} \frac{e^{-\beta_{1} J+\beta_{2} J}-e^{\beta_{1} J-\beta_{2} J}}{\left(e^{\beta_{1} J}+e^{-\beta_{1} J}\right)\left(e^{\beta_{2} J}+e^{-\beta_{2} J}\right)} .
$$

Proof. The existence statement is a consequence of the ergodicity of $P_{\mu}^{(L)}$, Proposition 2.1.

From this

$$
\begin{aligned}
\delta Q_{-L}= & 2 J\left[P _ { \mu } ^ { ( L ) } \left(\left|S_{-L}(1)-S_{-L+1}(1)\right|=2,\right.\right. \\
& \left.\left|S_{-L}(0)-S_{-L+1}(0)\right|=0, S_{-L+1}(1)=S_{-L+1}(0)\right) \\
& -P_{\mu}^{(L)}\left(\left|S_{-L}(1)-S_{-L+1}(1)\right|=0,\left|S_{-L}(0)-S_{-L+1}(0)\right|=2,\right. \\
& \left.\left.S_{-L+1}(1)=S_{-L+1}(1)=S_{-L+1}(0)\right)\right],
\end{aligned}
$$

which gives Eq. (2.12) by using Eq. (2.2) and Theorem 3.1. The evaluation of $\delta Q_{j}$, $j>-L$ is completely analogous.

Remark. The factor $\left(\frac{1}{2 L+1}\right) \frac{1}{4 L}$ in Eq. (2.12) instead of the expected $\frac{1}{2 L+1}$ is due to the fact that the evolution defined in Eq. (2.2) does not "scale well". In fact it 
converges locally to the trivial (identity) evolution as $L$ diverges, because of the factor $\frac{1}{2 L+1}$ in Eq. (2.2). When evaluating the stationary measure this circumstance does not play any role. However when we want to compute time evolved observables, as in Definition 2.2, we need to define correctly the time scale of our process. This is done in the following:

Definition 2.3. To every site $x \in[-L, L]$ we associate a time-line, on each of which we plot a Poisson process with mean one. For $x \in[-L+1, L-1]$ the mark reads " $a$ " (active) or " $p$ " (passive) with equal probability. For $x=L[-L]$ the mark is 1 with probability $\frac{e^{-\beta_{2} J}}{e^{\beta_{2} J}+e^{-\beta_{2} J}}\left[\frac{e^{-\beta_{1} J}}{e^{\beta_{1} J}+e^{-\beta_{1} J}}\right]$ or 0 with complementary probabilities.

This defines a stochastic continuous time evolution $P_{t}^{(L)}$ for the spin configurations according to the following rule. A spin $S_{x}, x \in[-L+1, L-1]$ can change only when an active mark " $a$ " appears at $x$. In that case the switch occurs whenever it does not change the total energy. The marks appearing at $x= \pm L$ determine the value of that spin as follows: when the mark 1 appears the spin at that time must become or remain of opposite sign from that of its nearest neighbor, and, when the mark 0 appears, of the same sign. It is easy to see that $\mu_{L}$ (of Proposition 2.1) is the only invariant measure and that the time evolution $P_{t}^{(L)}$ converges, locally, to an "infinite volume" time evolution $P_{t} . P_{t}$ can be explicitly constructed by simply considering Poisson time lines for each $x \in \mathbb{Z}$. On each line, independently as before, and with mean 1 , are put " $a$ " and " $p$ " marks with equal probability. Finally let $\delta Q_{j}(t)$ be the continuous time analogues of the $\delta Q_{j}(n)$ of Eq. (2.10), $\delta Q_{j}^{\text {(cont.) }}$ of $\delta Q_{j}$ as in Eq. (2.11). Then

Theorem 2.2. Almost surely with respect to the process $P_{t}^{(L)}$ with initial (invariant) measure $\mu_{L}$

$$
\delta Q_{j}^{\text {(cont.) }}=\frac{e^{\left(-\beta_{1}+\beta_{2}\right) J}-e^{\left(\beta_{1}-\beta_{2}\right) J}}{\left(e^{\beta_{1} J}+e^{-\beta_{1} J}\right)\left(e^{\beta_{2} J}+e^{-\beta_{2} J}\right)} \frac{2 J}{4 L}=-\delta Q_{L}^{(\text {cont. })} j=-L, \ldots, L-1 .
$$

As $L$ goes to infinity $L \delta Q_{-L}^{\text {(cont.) }}$ satisfies Fourier's law, Eq. (2.8), with $T(\xi)$ given as in Theorem 2.1 and with $K(T)=\frac{C(T)}{2}$.

Proof. It is completely analogous to that of Proposition 2.2 as far as equation (2.13) is concerned. The remainder follows by direct computation.

Remark. Notice that $\delta Q_{-L}^{\text {(cont.) }}$ correctly behaves like $L^{-1}$, when $L$ diverges. In Fourier's law, Eq. (2.8), there ought to be a factor $L^{-1}$, which is in fact absent because we rescaled the space variable by letting $\xi \in(-1,1)$ instead of $[-L, L]$ and we took $Q$ as $\lim L Q_{L}$. This is the reason why we had to consider in Theorem 2.2, $L \delta Q_{-L}^{\text {(cont.) }}$ rather than $\delta Q_{-L}^{\text {(cont.) }}$ itself.

In the remainder of the section we discuss the following model. Let $\mu_{0}$ be the semi-infinite DLR measure at temperature $T_{1}[6]$ on the left half space 
$(x=-1,-2, \ldots)$ and at temperature $T_{2}$ on the right one. Namely

$$
\begin{gathered}
\mu_{0}\left(S_{x} \mid S_{\{x\}}\right)=\exp \left[-\beta_{2,1} U\left(S_{x} \mid S_{x-1}, S_{x+1}\right)\right] \\
\left\{\exp \left[-\beta_{2,1} U\left(S_{x} \mid S_{x-1}, S_{x+1}\right)\right]+\exp \left[-\beta_{2,1} U\left(-S_{x} \mid S_{x-1}, S_{x+1}\right)\right]\right\}^{-1}, \\
x \geqq 1 \text { for } \beta_{2} \text { and } x \leqq-1 \text { for } \beta_{1}, \\
\mu_{0}\left(S_{0}\right)=\frac{1}{2},
\end{gathered}
$$

where

$$
\{x\}^{c}=\{y \in \mathbb{Z}: y \neq x\} .
$$

Equation (2.14) uniquely defines $\mu_{0}$, see [6].

Theorem 2.3. Let $\mu_{t}, t \geqq 0$, be the measure at time $t$ evolving according to $P_{t}$ (the "infinite volume" time evolution as in Definition 2.3). Then there exists $T(x)>0$, $x \in \mathbb{R}$, such that for every $x \in \mathbb{R}, f \in \mathcal{O}$

$$
\lim _{t \rightarrow \infty}\left[\mu_{t}\left(\tau_{[x \sqrt{t}]} f\right)-\mu^{(T(x))}(f)\right]=0 .
$$

See Definition 2.1 for $\mathcal{O}$ and $\tau_{[\cdot]} ; \mu^{(T(x))}$ is the DLR measure at temperature $T(x)$. Furthermore

$$
\begin{gathered}
T(x)=\frac{1}{K \beta(x \sqrt{t}, t)} \\
f(\beta(y, t))=f\left(\beta_{1}\right) \int_{0}^{\infty} \frac{d z}{\sqrt{2 \pi t}} \exp \left(-\frac{[z-y]^{2}}{2 t}\right)+f\left(\beta_{2}\right) \int_{-\infty}^{0} \frac{d z}{\sqrt{2 \pi t}} \exp \left(-\frac{[z-y]^{2}}{2 t}\right),
\end{gathered}
$$

where $f(\beta)$ is defined in Eq. (2.7a).

Proof. The proof is related to that of Liggett, see [7], and will be given in the next section, see the Remark after Lemma 3.1.

The relationship between the "temperature profile" $T(x, t)\left[=K^{-1} \beta(x, t)^{-1}\right]$ as in Theorem 2.3 and the heat conductivity, $K$, see Eq. (2.9), is given by the heat equation:

Theorem 2.4. Let $C(T), \hat{K}(T)$ be the specific heat and the heat conductivity coefficient; then the heat equation is

$$
C(T) \frac{\partial T(x, t)}{\partial t}=\frac{\partial}{\partial x}\left(\hat{K}(T(x, t)) \frac{\partial T(x, t)}{\partial x}\right) .
$$

For the Ising model

$$
C(T)=-J \frac{d}{d T} f(\beta), \quad \beta=\frac{1}{K T} .
$$

Then $T(x, t)$ satisfies Eq. (2.18) if

$$
\hat{K}(T)=\frac{1}{2} C(T),
$$


so that $\hat{K}(T)$ is the $K(T)$ of Eq. (2.9), with const. $=-\frac{1}{2} J$, as obtained in Theorem 2.2 . Proof. $-\frac{1}{J} E(T)$ is the left hand side of Eq. $(2.17) ; E(T)$ is increasing and invertible. Rewriting Eq. (2.18) in terms of $E(T)$ :

$$
\frac{\partial E}{\partial t}=\frac{\partial}{\partial x}\left(\frac{\hat{K}}{C} \frac{\partial E}{\partial x}\right)=\frac{1}{2} \frac{\partial^{2} E}{\partial x^{2}}
$$

because of Eq. (2.20). By Eq. (2.17) the thesis follows.

Remark. It is easy to see that if $\hat{K}(T)$ is smooth enough then it is uniquely determined by Eqs. (2.18) and (2.17) [as in Eq. (2.20)].

\section{Probability Estimates}

We introduce the random variables $n_{x}, x$ integer with values $n_{x}=0,1$, the former occurring if $S_{x}=S_{x+1}$, the latter when $S_{x} \neq S_{x+1}$. To any spin configuration $S$ in $\{-1,1\}^{2 L+1}$ there corresponds a "particle configuration" $\left\{n_{x}, x=-L, \ldots, L-1\right\}$. The configurations $S,-S$ (obtained from the other by flipping each spin) give rise to the same $\left\{n_{x}\right\}$. The following quite obvious proposition links the measure $\mu_{L}$, introduced in Proposition 2.1, to its relativization to the $\left\{n_{x}\right\}$ variables:

Proposition 3.1. Let $\mu_{L}$ be given by Proposition 2.1. Let

$$
\mathscr{R}:\{-1,+1\}^{2 L+1} \rightarrow\{-1,+1\}^{2 L+1}(\mathscr{R} S)_{x}=-S_{x} \forall x,
$$

then

$$
\begin{aligned}
& \mu_{L}(S)=\mu_{L}(\mathscr{R} S) \\
& \mu_{L}(S)=\frac{1}{2} \mu_{L}(n(S)),
\end{aligned}
$$

where $n(S)$ is the "particle configuration" corresponding to $S$.

Proof. Let $\hat{\mathscr{R}}$ act on the space of probability measures over $\{-1,+1\}^{2 L+1}$

$$
(\hat{\mathscr{R}} v)(S)=v(\mathscr{R} S), \hat{\mathscr{R}}^{2}=1 .
$$

For $P$ as in Eq. (2.2)

$$
\begin{aligned}
& \hat{\mathscr{R}} P \hat{\mathscr{R}}=P \\
& P \hat{\mathscr{R}} \mu_{L}=\hat{\mathscr{R}}(\hat{\mathscr{R}} P \hat{\mathscr{R}}) \mu_{L}=\hat{\mathscr{R}} P \mu_{L}=\hat{\mathscr{R}} \mu_{L}
\end{aligned}
$$

and because of the uniqueness of $\mu_{L}$ (see Proposition 2.1) this concludes the proof.

Proposition 3.1 allows us to study the measure $\mu_{L}$ regarded as a measure on the $\sigma$-algebra generated by the $\left\{n_{x}\right\}$; in the sequel we will denote it by the same symbol, $\mu_{L}$. 
The stochastic evolution Eq. (2.2) in the particle representation is also Markov with transition probability:

$$
\begin{aligned}
P\left(n, n^{\prime}\right)= & \sum_{x=-L}^{L-2} \frac{1}{2 L+1} \frac{\delta\left(n_{x}^{\prime}+n_{x+1}^{\prime}-n_{x}-n_{x+1}\right)}{\#\left(n_{x}+n_{x+1}\right)} \prod_{y \neq x, x+1} \delta\left(n_{y}-n_{y}^{\prime}\right) \\
& +\frac{1}{2 L+1} \frac{e^{\beta_{1} J\left(1-2 n_{-L}\right)}}{e^{-\beta_{1} J}+e^{\beta_{1} J}} \prod_{y>-L} \delta\left(n_{y}-n_{y}^{\prime}\right) \\
& +\frac{1}{2 L+1} \frac{e^{\beta_{2} J\left(1-2 n_{L}\right)}}{e^{-\beta_{1} J}+e^{\beta_{1} J}} \prod_{y<L-1} \delta\left(n_{y}-n_{y}^{\prime}\right), \\
& \#\left(n_{x}+n_{x+1}\right)=\operatorname{Card}\left(\left(n_{x}^{\prime}, n_{x+1}^{\prime}\right): n_{x}^{\prime}+n_{x+1}^{\prime}=n_{x}+n_{x+1}\right) .
\end{aligned}
$$

This evolution represents particles moving only one at a time to neighboring sites, if empty. At the extremal sites particles can be created or destroyed independently and with rates determined by $\beta_{1}, \beta_{2}$ as in Eq. (3.1) ${ }^{2}$. As in Proposition 2.1 the Markov chain with transition probability $P\left(n, n^{\prime}\right)$ is irreducible aperiodic and there is only one measure, $\mu_{L}$, which is $P$-invariant.

To study the asymptotic behavior of $\mu_{L}$ as $L$ diverges we use a very simple and powerful "graphical representation" of the correlation functions, see [8]. To avoid notational complications we will assume that the particle sites are $-L, \ldots, L$ (in the limit $L$ going to infinity this turns out to be irrelevant).

We begin by introducing the analogue of the process defined in Definition 2.3, but use the "particle language". We will consider possible variations of the probability distribution governing the "thermalization" of the spins close to the boundaries. The physical reason for this is given in the Introduction and in the Concluding Remarks of Sect. 4 ; we treat here the more general case because our formalism would not change much anyhow.

Definition 3.1. We consider in this definition $L 0 \leqq m \leqq L$ fixed. $m$ will be kept the same as $L$ goes to infinity and $[-L,-L+m],[L-m, L]$ are the "sites" where the rate is going to be changed. So given $L, m$ we introduce a realization of the continuous-time Markov chain which has the same invariant measure $\mu_{L}$ as the discrete time analogue and which will enable us to "reverse the time" in an appropriate sense. To each pair of nearest neighborhood sites we associate a timeline and on each of these lines we plot a Poisson point process. This process has mean 1 for all the lines between the sites $[-L+m, L-m]$. For the other lines (it is also convenient to draw a line between $-L-1,-L$ and another one between $L, L+1)$ we plot the marks with means $0<\gamma_{i}<\infty$ : namely in the line $L-m+i$, $L-m+i+1$ the mean is $\gamma_{i}, i=0, \ldots, m$ : Analogous is the procedure for the lines between $-L-1,-L+m$. Each mark referring to internal lines can read " $a$ " (active) or " $p$ " (passive). The probability for the " $a$ " and " $p$ " is the same. On the external lines the mark read " $b$ " (birth) with probability

$$
\frac{e^{-\beta_{1}}}{e^{\beta_{1}}+e^{-\beta_{1}}} \text { at the left } \frac{e^{-\beta_{2}}}{e^{\beta_{2}}+{ }^{-\beta_{2}}} \text { at the right }
$$

and " $d$ " (death) with the complementary probabilities.

2 This system is considered in [14] and the stationary measure is explicitly given if particles are only created at $-L$ (no particle's death at $-L$ ) and only destroyed at $L$ (no particle's birth at $+L$ ) 


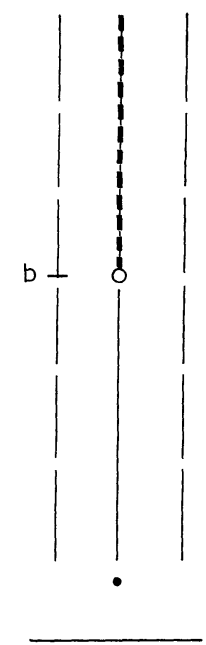

time line

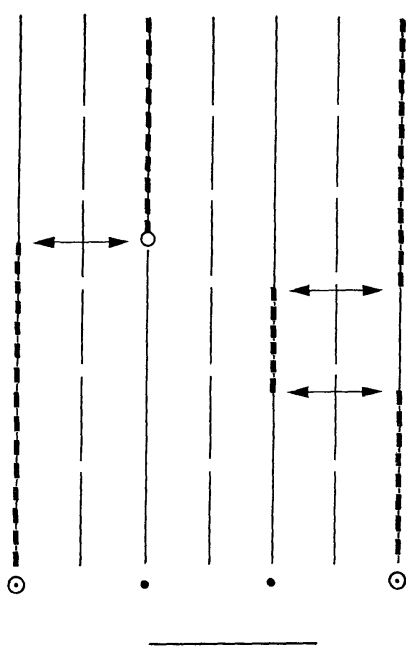

position
$t=T$

I

1

1

1

$+b$

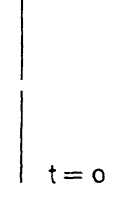

Fig. 1

All these Poisson processes are independent. For each realization we have a trajectory of particles according to the following procedure. Given an initial configuration of particles (plotted as "0" in Fig. 1) we switch the values at the neighbouring sites when an " $a$ " mark (arrow in the figure) occurs, and at the boundaries we create (destroy) a particle according to the mark " $b$ " (" $d$ ") if they fail to be already existing (absent). If a particle is already at site $L$ when a sign " $b$ " occurs, the particle is not affected (if the site was void and a " $d$ " mark occurs, it remains empty).

In order to recover the Markov chain of Proposition 3.1 look at the discrete time skeleton, obtained by observing the process only at the instants when a mark appears on some of the time-lines (with probability one, two marks never appear at the same time). This Markov evolution has transition probability given by equation (3.1) when we choose $m=0$ and the two $\gamma$ 's both equal to 1 .

Remark. This realization is important to us because all the randomness is concentrated in the laws of the point-process (Poisson) and of the marks (independent, equal probability ...). Given this random configuration, the evolution is deterministic and we can use one-one correspondence. Furthermore if we wish to focus on a certain aspect of the evolution (e.g. the evolution of one ghost particle among others, see Definition 3.2) and make a statement dealing with this aspect only, we need only consider the "law" of this part of the process. For instance we will see that "a ghost particle performs a random walk, which is known to escape from any fixed bounded region almost surely," which means that those random configurations such that this happens have probability one.

Eventually we must remark that the continuous time setting is not necessary for reversing the time. It will only be used for the renormalization procedure in Lemma 3.3.

Definition 3.2. By using the above graphical representation it appears that if we want to know what is the value of the process at a time $t$ and at a certain number 
of sites (correlation functions of these sites at time $t$ ) we just need to read backwards. Each site will then be "moving" back according to the arrows in the graphical representation, and in order to avoid notational confusion we will call the trajectory of the site "the trajectory of the ghost particle." Namely every site involved in the correlation function becomes a "ghost particle" whose trajectory is determined by the above graphical rule. The probability that several sites are occupied or empty at time $t$, given an initial configuration of "real" particles at time 0 , is the probability that the corresponding ghost particles are present, $\eta_{x}=1$, or absent $\eta_{x}=0$. A particle is present at times $t$ at the site $x$ iff one of the following events occurs:

i) the ghost particle reads a mark at one of the extremal lines before $t$ (going backwards) and this is $b$ (it is meaningful in what follows to consider the trajectory of the ghost particle to finish when it reads a " $b$ " or " $d$ " mark)

ii) the "ghost particle never reads a " $d$ " or " $b$ " mark before $t$ and goes to an occupied site at time 0 , (the bottom of the diagram).

Because of the special property of the Poisson point processes, namely that read backwards they are still Poisson point processes, we note that the ghost particles also perform a continuous time Markov evolution. This is a simple exclusion process with two additional sites at $\pm(L+1)$ where they are "absorbed" and read their final sentence, " $b$ " or " $d$ ". The limiting distribution of the particles as $t$ diverges is the unique invariant measure of the process, and therefore is $\mu_{L}$ (the same as of Sect. 2 for the special choice of $m=0$ and $\gamma=1$, as noted in Definition 3.1). This is so because the probability that a particle survives [i.e. it never gets to $\pm(L+1)$ before $t]$ goes to zero as $t$ diverges.

It is convenient to give notation for the above quantities: we denote by $(\Omega, \mathscr{P})$ the probability space which realizes the Poisson independent processes with their marks, $\eta_{x}$ are the occupation number variables ( 0 or 1$)$ for the ghost particles, $X(t)$ their trajectories.

It is very easy to study the one-body correlation functions. We will then analyze the two-point correlations, again quite explicitly, and finally we will need to use a more abstract argument for the $n$-body correlations.

It is easy to see that a single ghost-particle, when observed at the instants when it moves, performs (in law) a simple Markov chain with transition probability $p(x, x+1)=p(x, x-1)=1 / 2$ if $-L+m+1 \leqq x \leqq L-m-1$ and

$$
\begin{aligned}
p(x, x+1) & =\frac{\gamma_{i-1}}{\gamma_{i-1}+\gamma_{i}}, p(x, x-1)=\frac{\gamma_{i}}{\gamma_{i-1}+\gamma_{i}}, \\
\gamma_{-1} & =1
\end{aligned}
$$

if $x=L-m+i$ and

$$
p(L, L+1)=\frac{2 \gamma_{m-1}}{2 \gamma_{m-1}+\gamma_{m}}=1-p(L, L-1),
$$

and analogously on the other side.

Definition 3.3. We will denote by $P_{L}^{*}$ the law of the Markov chain with transition probability defined above. 
Theorem 3.1. Let $\xi \in(-1,1)$ and let $x$ be fixed. Then

$$
\lim _{L \rightarrow \infty} \mu_{L}\left(n_{[\xi L]+x}\right)=\frac{1}{2}(1-\xi) \tilde{f}\left(\beta_{1}\right)+\frac{1}{2}(1+\xi) \tilde{f}\left(\beta_{2}\right),
$$

where

$$
\tilde{f}(\beta)=\frac{e^{-\beta J}}{e^{\beta J}+e^{-\beta J}} .
$$

In particular if $m=0, \gamma_{ \pm}=1$ (see Definition 3.1) and $y \in[-L, L-1]$, we are in the original case considered in Sect. 2 and

$$
\mu_{L}\left(n_{y}=1\right)=\left(\frac{2 L-1}{4 L}-\frac{y}{2 L}\right) \tilde{f}\left(\beta_{1}\right)+\left(\frac{2 L+1}{4 L}+\frac{y}{2 L}\right) \tilde{f}\left(\beta_{2}\right) .
$$

Proof. Since the values of the marks in $(\Omega, \mathscr{P})$ are independent of the rest of the process we have

$$
\mu_{L}\left(n_{y}=1\right)=\tilde{f}\left(\beta_{1}\right) P_{L}^{*}(X(T)=-L-1)+\tilde{f}\left(\beta_{2}\right) P_{L}^{*}(X(T)=L),
$$

where $P_{L}^{*}$ is given in Definition 3.3 and $T$ is the first moment the particle reaches either $-L-1$ or $L$. To estimate the exit probabilities in Eq. (3.5) we remark that they are harmonic functions [5] and that they can be easily computed in an explicit way when $m=0$. From this Eq. (3.4). To get Eq. (3.3) we introduce a new stopping time $T^{\prime}$ which is the first time the random walk gets to $\pm(L-m)$. Up to this time the random walk is $1 / 21 / 2$ and so

$$
P_{L}^{*}\left(X\left(T^{\prime}\right)=a(L-m)\right)=\left(\frac{1}{2}+\frac{a y}{2(L-m)}\right), \quad a= \pm 1 .
$$

On the other hand the probability starting from $a(L-m)$ to get to $a(L+1)$ goes to 1 when $L$ goes to infinity and from Eq. (3.5) and (3.6) we prove Eq. (3.3). Theorem 3.1 is therefore proved.

Our next goal is to prove the following

Theorem 3.2. For every $K>0, x_{1}, \ldots, x_{K} \in \mathbb{Z}, n_{1}, \ldots n_{K} \in\{0,1\}^{K}$ and $\xi \in(-1,1)$

$$
\lim _{L \rightarrow \infty} \mu_{L}\left(n_{x_{1}+[\xi L]}=n_{1}, \ldots, n_{x_{K}+[\xi L]}=n_{K}\right)=\lim _{L \rightarrow \infty} \prod_{i=1}^{K} \mu_{L}\left(n_{x_{i+[\xi L]}}=n_{i}\right)
$$

which is given by Eq. (3.3).

Theorem 3.1 and 3.2 yield the

Corollary. For every $\xi \in(-1,1), v_{\xi}^{(L)}$, see Eq. (2.6), converges to the Gibbs measure at temperature $T(\xi)=(K \beta(\xi))^{-1}$ given by

$$
f(\beta(\xi))=\frac{1}{2} f\left(\beta_{1}\right)(1-\xi)+\frac{1}{2} f\left(\beta_{2}\right)(1+\xi),
$$

where $f$ is defined by Eq. (2.7a).

We first prove Theorem 3.2 with $K=2$ and to do so we introduce a definition and some lemmas. 
Definition 3.4. We consider the trajectories of two particles starting at $x$ and $y$. Sometimes we will consider this as a trajectory in the space $\mathbb{Z}^{2}$ for the point $(X(t)$, $Y(t))$.

We will denote by $P_{L}$ the law of the corresponding Markov chain. It differs from the law of two independent random walks in continuous time when

i) the two particles are in nearest neighborhood sites

ii) at least one of the two particles is in the region $[-L-1,-L+m]$ and $[L-m, L+1]$

iii) after a particle has reached $\pm(L+1)$.

In the sequel we will somehow try to reduce to the independent case. A first step is to introduce the process $P_{(f)}$, where $f$ stands for free of the "boundary conditions". Namely $L$ is taken to be infinite so that $P_{(f)}$ differs from the independent $1 / 2,1 / 2$ random walk only for the condition i) above.

We now introduce some shorthand notation:

$$
\begin{aligned}
\partial \Gamma= & \left\{(x, y) \in \mathbb{Z}^{2} \mid \sup (|x|,|y|)=L-m\right\}, \\
a= & \left(a_{1}, a_{2}\right), \quad a_{i}= \pm 1, \\
\{a\}= & \left\{(X(t), Y(t))_{t \in \mathbb{R}_{+}} \mid \text {first exit of } X(t)\right. \text { occurs at } \\
& \left.a_{1}(L+1) \text { and first exit of } Y(t) \text { occurs at } a_{2}(L+1)\right\}, \\
T= & \text { first time a particle arrives at } \partial \Gamma, \\
H(x, y)= & P_{L}(\{a\} \mid(X(0), Y(0)=(x, y)) .
\end{aligned}
$$

Even though this does not appear explicitly, notice that the above defined quantities do depend on $L$. $H(x, y)$ will only be considered as defined on $\partial \Gamma$. For $\varepsilon>0$ let:

$$
\begin{aligned}
D(x, y) & =1_{\{|x-y| \leqq \varepsilon L\}} \\
& =\text { characteristic function of the set }\{|x-y| \leqq \varepsilon L\} \text { in } \mathbb{Z}^{2}
\end{aligned}
$$

and

$$
\begin{aligned}
h(x, y)= & \left(\frac{1}{2}+\frac{a_{1} x}{2(L+1)}\right) \delta\left(\frac{y}{L-m}-a_{2}\right) \\
& +\left(\frac{1}{2}+\frac{a_{2} y}{2(L+1)}\right) \delta\left(\frac{x}{L-m}-a_{1}\right) .
\end{aligned}
$$

We then have the obvious

Lemma 3.1. With the notation above

$$
\left|P_{L}[\{a\} \mid(X(0), Y(0))=(x, y)]-E_{(f)}\left(h^{T}\right)\right| \leqq 2 E_{(f)}\left(D^{T}\right)+\left|E_{(f)}\left[\left(h^{T}-H^{T}\right)\left(1-D^{T}\right)\right]\right|,
$$

where the superscript T means that the function has to be evaluated at the position at the random time $T$ (defined in Eq. (3.12)); $E_{(f)}$ is the expectation for the process $P_{(f)}$ starting at $(x, y)$. 
Proof. We simply note that

$$
P_{L}[\{a\} \mid(X(0), Y(0))=(x, y)]=E_{(f)}\left(H^{T}\right) .
$$

Equation (3.16) is then obtained straightforwardly.

We are going to show that the right hand side of Eq. (3.16) is vanishingly small for large $L$ and so it will remain to estimate $E_{(f)}\left(h^{T}\right)$.

Lemma 3.2. Let $(x, y) \in \partial \Gamma$ (see Eq. (3.9)). Then

$$
\lim _{L \rightarrow \infty} \sup _{(x, y) \in \partial \Gamma} \mid(h(x, y)-H(x, y))(1-D(x, y) \mid=0,
$$

where $h, H$ and $D$ are defined respectively in Eqs. (3.15), (3.13), (3.14). As a consequence the last term in Eq. (3.16) goes to zero as L goes to infinity for any fixed $\varepsilon>0$.

Proof. The idea of the proof is the following. $H(x, y)$ is the probability that $x$ exits at $a_{1}(L+1)$ and $y$ at $a_{2}(L+1)$. But one of the two particles, let us say $x$, is close to the boundary i.e. at distance $m+1$ from $L+1$ (for example). The other one is very far away, otherwise : $1-D=0$. The $x$-particle will reach $L+1$ before it gets "close" to the $y$-particle, with very large probability. Therefore the two particles move independently and so $H$ and $h$ are equal (in the limit $L$ going to infinity). In fact by using the same argument as in the proof of Theorem 3.1 it is enough to prove that one of the two particles reaches $\pm(L+1)$ before the two become nearest neighbors. To fix the ideas, let us assume as before that $X=L-m$ and that $|y-x| \geqq \varepsilon L$. Let $\hat{T}$ be the exit time at $L+1$ for a single particle which is initially at $L-m$ and performs a $(1 / 2,1 / 2)$ random walk when its position is $x<L-m$ and with the probability specified in Def. 3.3 when $L-m \leqq x \leqq L+1$. It is easy to see that $\hat{T}$ is almost surely finite. Then for every $\delta>0$ there is $T$ such that the probability that $\hat{T}>T$ is less than $\delta$. Furthermore there exists $d \in \mathbb{Z}^{+}$such that a $(1 / 2,1 / 2)$ random walk does not get further than $d$ within time $T$, with probability larger than $1-\delta$. When $L$ is larger than $\frac{2 d}{\varepsilon}$, with probability larger than $1-3 \delta$, the $x$-particles reach $L+1$ before the $x$ and $y$ particles become nearest neighbors.

To evaluate $E_{(f)}\left(D^{T}\right)$ and $E_{(f)}\left(h^{T}\right)$ in Eq. (3.16) we use a renormalization procedure, by shrinking the lattice by $\frac{1}{L-m}$ and by speeding up the time by a factor $2 L^{2}$. In this way we are considering a sequence of processes $P_{(f)}^{(L)}$ in the same trajectory space and

Lemma 3.3. $P_{(f)}^{(L)}$ converges in distribution to $P_{(b)}$, the Brownian motion in two dimensions.

Before proving Lemma 3.3 we note that the convergence in distribution will (see Lemma 3.4) imply the weak convergence on a class of functions which includes both $D^{T}$ and $h^{T}$, more precisely these functions written in the renormalized variables.

Proof of Lemma 3.3. The trajectory space is $D\left(\mathbb{R}_{+}, \mathbb{R}^{2}\right)$, see for instance [10] Chap. 5. The measure $P_{(f)}^{(L)}$ are characterized by Eq. (3.18) below. Call $\xi_{t}$ the 
element in $D\left(\mathbb{R}_{+}, \mathbb{R}^{2}\right)$ and suppose

i) $\Phi$ is a bounded function measurable with respect to the $\sigma$-algebra generated by the process up to time $s>0$;

ii) $f$ is a real $C^{3}$ function on $\mathbb{R}^{2}$ with compact support. Then $\left(E_{(f)}^{(L)}\right.$ is the expectation w.r.t. $\left.P_{(f)}^{(L)}\right)$

$$
E_{(f)}^{(L)}\left(\Phi\left[f\left(\xi_{t}\right)-f\left(\xi_{s}\right)\right]\right)=E_{(f)}^{(L)}\left(\Phi \cdot \int_{S}^{t} A^{L} f\left(\xi_{u}\right) d u\right) \forall t>s>0,
$$

where (for notational simplicity in the following formulas the shrinking factor for the space variable is considered to be $L$ rather than $L-m)$ :

$$
\begin{aligned}
A^{L} f(x, y)= & L^{2}\left[f\left(x+\frac{1}{L}, y\right)+f\left(x, y+\frac{1}{L}\right)+f\left(x-\frac{1}{L}, y\right)\right. \\
& \left.+f\left(x, y-\frac{1}{L}\right)-4 f(x, y)\right], \text { if }|x-y|>\frac{1}{L}, \\
A^{L} f(x, y)= & L^{2}\left[f\left(x+\frac{1}{L}, x\right)+f\left(x-\frac{1}{L}, x+\frac{1}{L}\right)+f\left(x, x+\frac{2}{L}\right)\right. \\
& \left.-3 f\left(x, x+\frac{1}{L}\right)\right], \quad \text { if } \quad y=x+\frac{1}{L}, \\
A^{L} f(x, y)= & L^{2}\left[f\left(x-\frac{1}{L}, x\right)+f\left(x, x-\frac{2}{L}\right)+f\left(x+\frac{1}{L}, x-\frac{1}{L}\right)\right. \\
& \left.-3 f\left(x, x-\frac{1}{L}\right)\right], \quad \text { if } \quad y=x-\frac{1}{L} .
\end{aligned}
$$

For each fixed $f$

$$
\begin{aligned}
& \left(A^{L} f\right)(x, y)=\Delta f(x, y)+0\left(\frac{1}{L}\right)|x-y|>\frac{1}{L} \\
& \left(A^{L} f\right)(x, y)=\left(\Delta f(x, y)-\frac{\partial^{2} f}{\partial x \partial y}(x, y)\right)+0\left(\frac{1}{L}\right)|x-y|=\frac{1}{L} .
\end{aligned}
$$

Therefore the right hand side of Eq. (3.18) is almost equal to

$$
E_{(f)}^{L}\left(\Phi \cdot \int_{s}^{t} \Delta f\left(\xi_{u}\right) d u\right)
$$

except for a term of size $0(1 / L)$ and another bounded by

$$
\begin{aligned}
& (\sup \Phi) \cdot E_{(f)}^{L}\left(\int_{0}^{t} 1_{(|x-y|=1) / L}\left(\xi_{u}\right) d u\right), \\
& 1_{(\cdot)}\left(\xi_{u}\right)=\text { ch. function that } \xi_{u} \in(\cdot) .
\end{aligned}
$$

This represents the amount of time the process spends in the region $\left\{(x, y):|x-y|=\frac{1}{L}\right\}$. To estimate it we go back to the unaccelerated free Markov 
chain. When outside of $\{|x-y|=1\}$ the chain waits a time $U$ before entering this set and $U$ has the same law as the waiting time for a simple one-dimensional random walk to reach zero starting from $|x-y|-1$. When in the set $\{|x-y|=1\}$ the chain remains a "geometric" time inside. So the amount of time spent in $\{|x-y|=1\}$ between $L^{2} s$ and $L^{2} t$ is of order $L$, hence of order $1 / L$ after renormalization (up to a factor $|t-\varepsilon|$ ). On the other hand, the family $P_{(f)}^{L}$ is weakly compact in the Skorokhod topology $([11])$ and therefore any weak limit of $P_{(f)}^{L}$ satisfies

$$
E\left(\Phi \cdot \int_{s}^{t} f\left(\xi_{u}\right) d u\right)=E\left(\Phi \cdot\left[f\left(\xi_{t}\right)-f\left(\xi_{s}\right)\right]\right)
$$

Equation (3.23) read for $\Phi, f, s, t$ as in Eq. (3.18) completely characterizes the process which is therefore the independent Brownian motion [10].

Remark. The above result remains unchanged even if we consider the manyparticles case. Therefore we have proven Eq. (2.15). Since Eqs. (2.17) and (2.16) are standard estimates for single particle random walks, Theorem 2.3 is also proven.

Lemma 3.4. Let $\partial \tilde{\Gamma}$ be the boundary of the unit square in $\mathbb{R}^{2}, \tilde{D}$ the characteristic function of the set

$$
\begin{aligned}
\{(x, y) \in \partial \tilde{\Gamma}|| x-y \mid \leqq & \varepsilon\}, \\
\tilde{h}(x, y)= & \frac{1}{2}\left(1+a_{1} x\right) \delta\left(y-a_{2}\right), \\
& +\frac{1}{2}\left(1+a_{2} y\right) \delta\left(x-a_{1}\right),
\end{aligned}
$$

$\tilde{T}$ and the stopping time at $\partial \tilde{\Gamma}, \tilde{D} \tilde{T}, \tilde{h}^{\tilde{T}}$ are the random variables obtained by computing $\tilde{D}$ and $\tilde{h}$ at the random time $\tilde{T}$. (These definitions are the renormalized analogues of those for $\partial \Gamma, D, h)$. Then

$$
\begin{aligned}
& \lim _{L \rightarrow \infty} E_{(f)}\left(D^{T}\right)=E_{(b)}\left(\tilde{D}^{\tilde{T}}\right) \\
& \lim _{L \rightarrow \infty} E_{(f)}\left(h^{T}\right)=E_{(b)}\left(\tilde{h}^{\tilde{T}}\right) .
\end{aligned}
$$

Proof. The function $D^{T}$ becomes $\tilde{D}^{\tilde{T}}$ after the scale changes. This is not continuous in the Skorokhod topology [10] but the set of its discontinuities has zero-measure with respect to $P_{(b)}$. (This is so because the discontinuities arise from trajectories $\xi_{t}$ which are either tangent to $\partial \tilde{\Gamma}$ or which cross $\partial \tilde{\Gamma}$ at the points $|x-y|=\varepsilon$.)

Equation (3.25) is then a consequence of Lemma 3.3 via Theorem 5.5 of [10]. For Eq. (3.26) the argument is very similar. The only remark is that $h^{T}$ when rescaled is not $\tilde{h}^{\tilde{T}}$. The difference can however be uniformly estimated by something vanishingly small as $L$ diverges.

Proof of Theorem 3.2 for the case $K=2$. This is a straightforward consequence of Eq. (3.16), of Lemma 3.2 and of Eqs. (3.25) and (3.26).

Remark. Of course one would guess that this result holds for $K>2$ (in Theorem 3.2). However the functions which play the role of $H$ do depend on $L$ and we lack 
information on the uniformity of their convergence (which we exploited in the case $K=2$ in Lemma 3.4).

To prove Theorem 3.2, we will first establish an asymptotic exchangeability for our stationary measures $\mu_{L}$, then we will use De Finetti's theorem and the already obtained factorization of the two body correlations: this procedure is very similar to that used in [12]. In fact the proof of the following Lemma 3.5 could also be obtained in close analogy with that of Theorems 1, 2 of Spitzer [12].

Lemma 3.5. Let $\sigma$ be any permutation of the set of indices $\{1, \ldots, K\}$ then:

$$
\begin{aligned}
& \lim _{L \rightarrow \infty} \mu_{L}\left(n_{x_{1}+[x L]}\right.\left.=n_{1}, \ldots, n_{x_{K}+[x L]}=n_{K}\right), \\
&\left.-\mu_{L}\left(n_{x_{1}+[x L]}=n_{\sigma(1)}, \ldots, n_{x_{K}+[x L]}=n_{\sigma(K)}\right)\right]=0,
\end{aligned}
$$

where $n_{1}, \ldots, n_{K} \in\{0,1\}^{K}$.

Proof. We have to go back to the original Poisson-point process, (Definition 3.2). Given an initial configuration of "ghost particles", focus on two of them. Then the following transformation $S$ on $\Omega$ (the space of realizations of the process) is defined, see Fig. 2.

On the set $\mathscr{G}: \mathscr{G}$ is such that there is a first time when the position of the two particles are nearest neighbour and there is a mark in the line connecting them. $S$ acts by changing this mark (from " $a$ " to " $p$ " or vice versa).

On the complement of $\mathscr{G}, S$ acts as the identity. It is quite easy to see that the transformation $S$ does not change the probability. The essential point is that on $\mathscr{G}$ the transformation $S$ is such that the trajectories for all the particles different from $i$ or $j$ are the same for $\omega$ or $S(\omega)$, and are exchanged for $i$ and $j$ after this fatal moment. In particular this yields:

$$
\begin{aligned}
\mathscr{P}\left(\eta_{x_{1}}\right. & \left.=n_{1}, \ldots, \eta_{x_{l}}=n_{i}, \ldots, \eta_{x_{j}}=n_{j}, \ldots, \eta_{x_{K}}=n_{K}, \omega \in \mathscr{G}\right) \\
& =\mathscr{P}\left(\eta_{x_{1}}=n_{1}, \ldots, \eta_{x_{\imath}}=n_{j}, \ldots, \eta_{x_{j}}=n_{i}, \ldots, \eta_{x_{K}}=n_{K}, \omega \in \mathscr{G}\right) .
\end{aligned}
$$

$\mathscr{P}(\mathscr{G})$ converges to 1 as $L$ diverges. This is so because of the following two facts. First, each time the particles are nearest neighbour there is a probability $1 / 2$ that a mark between these positions appears before the two particles separate. Secondly, when separated the two particles move according to independent simple random walks, and move at least $(1-|\xi|) L$ times before one of them is absorbed at the boundary. A simple random walk comes back to zero more than $N^{\beta}$-times $(\beta<1 / 2)$ before $N$ with probability tending to 1 as $N$ diverges. Hence we have proved the claim for any transposition of the indices, and therefore for any permutation, since this can be written as a product of transpositions.

From Lemma 3.5 it follows that the weak limit of any convergent subsequence of $\mu_{L}$ 's is exchangeable and therefore by De Finetti's theorem it is a mixture of Bernouilli measures. From Theorem 3.2 with $K=2$, which we have already proven, the 2-body correlation function factorizes and this implies that the weak limit is Bernouilli itself. For, if $v$ is the law of the mixture, we have

$$
\int_{0}^{1} p^{2} v(d p)=\left(\int_{0}^{1} p v(d p)\right)^{2}
$$



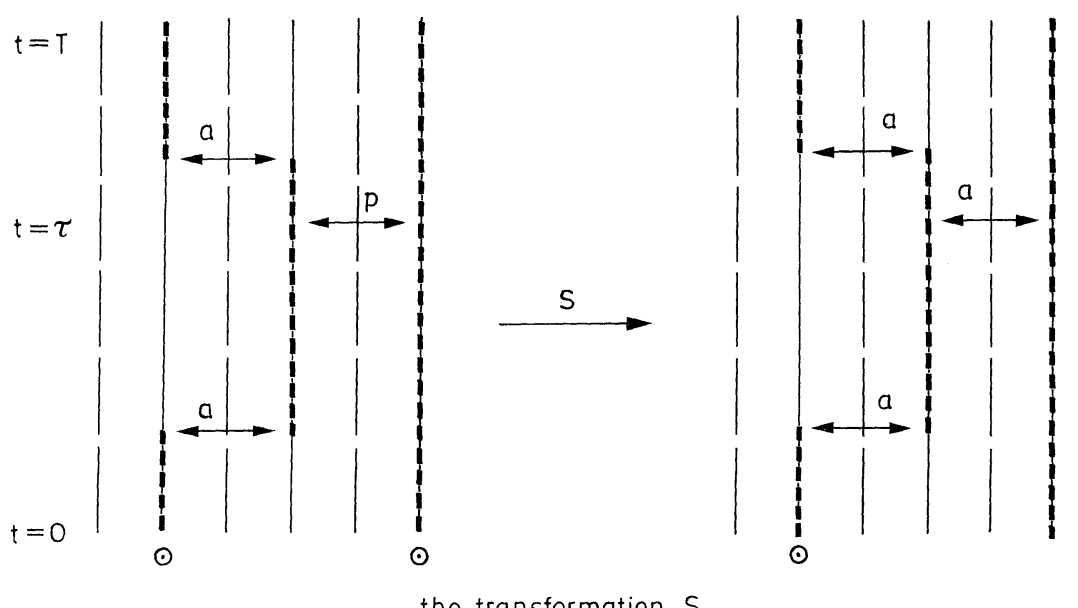

the transformation $S$

Fig. 2

and strict convexity of $p^{2}$ forces $v(d p)$ to be a Dirac mass. The parameter of the Bernoulli measure is the same for each subsequence by Theorem 3.1 and so Theorem 3.2 is proven.

\section{Concluding Remarks}

There is some arbitrariness in the choice of the size of the subsystems for which it is assumed that "local equilibrium" holds: namely in Eq. (2.2) the choice was that only a single spin at a time could change. Analogously we could have assumed that a block of $m$ spins at a time is "thermalized", as explained in the introduction (only changes which keep the same energy are allowed). As a consequence the measure $\mu_{L}$ changes but the effect disappears as $L$ diverges.

Notice that in Sect. 3 we have actually proven a stronger result than that stated in Theorem 2.1. Namely that if we change the rate of the thermalization atx $\in[-L, L]$ by keeping it uniform between $-L+m, L-m, m$ fixed when $L$ diverges, then the stationary measure $\mu_{L}$ changes but in the limit the change disappears.

It is physically conjectured [2] that the relevant information on the heat transport phenomena should be found in the $1 / L$ asymptotic correction to the measure $\mu_{L}$ with respect to its limiting value. In our case it ought to be possible to prove rigorously the existence of such a state.

It should be interesting to study different spin systems (i.e. more general interactions, higher dimensional cases) than the model we have treated here and to check whether analogous results hold. The same definition of local equilibrium and stationary measure under the stochastic evolution could be applied to systems of oscillators for which a deterministic dynamics exists. Is then the measure $\mu_{L}$ stationary under the deterministic dynamics for a finite time up to order $1 / L$ (included)? The leading term for $\mu_{L}$ should go to the Gibbs state (if our results 
extend to this case) and so it would be automatically invariant and the problem remains if the correction in $L^{-1}$ is also stationary. It would also be interesting to compare $\mu_{L}$ with the measure $\tilde{\mu}_{L}$ defined as the invariant measure for the dynamics with stochastic boundaries like that introduced in [2] for the particles' case. Again the guess is that the two measures are the same up to order $1 / L$.

Another question: is it possible to give some definition of local equilibrium, like the one we used here, if continuous particle systems are considered? The physical problem is that in this case mass transport phenomena are present.

Acknowledgements. We are deeply indebted to M. Roussignol and C. Cocozza for many helpful discussions and suggestions. One of us (A.G.) wishes to thank the "Laboratoire de Probabilité de l'Université Paris VI" for its kind hospitality. Two of us (C.M., E.P.) acknowledge very kind hospitality at IHES. We are very much indebted to the referee of C.M.P. for a careful and critical reading of the manuscript.

\section{References}

1. Spohn, H., Lebowitz, J.L.: Commun. Math. Phys. 54, 97 (1977);

Lebowitz, J.L., Spohn, H.: J. Stat. Phys. (to appear);

Aizenmann, M., Spohn, H.: J. Stat. Phys. 21, 23 (1979);

O’Connor, A.J., Lebowitz, J.L. : J. Math. Phys. 15, 692-703 (1974)

2. Goldstein, S., Lebowitz, J.L., Presutti, E.: Mechanical systems with stochastic boundaries. In: Lecture at the Colloqium on "Random fields: rigorous results in statistical mechanics and quantum field theory". Esztergon, Hungary (1979)

3. Georgii, H.O.: "Canonical Gibbs measures. In: Lecture Notes in Mathematics, Vol. 760. Berlin, Heidelberg, New York: Springer 1979

4. Ruelle, D.: Statistical mechanics. New York: Benjamin 1968

5. Revuz, D.: Markov chains. North Holland: American Elsevier 1975

6. Ruelle, D.: Commun. Math. Phys. 9, 267 (1968)

7. Liggett, T.: The stochastic evolution of infinite systems of interacting particles. In: Lecture Notes in Mathematics, Vol. 598, pp. 188-248. Berlin, Heidelberg, New York: Springer 1976

8. Bertein, F., Galves, A.: Z. Wahrsch. Verw. Gebiete 41, 73-85 (1977);

Bertein, F., Galves, A.: C. R. Acad. Sci. 285, 10 (1977)

Griffeath, D.: Ann. Prob. 6, 379-387 (1978);

Harris, T. : Ann. Prob. 6, 355-378 (1978);

Griffeath, D. : Graphical treatment of associate process. Séminaire de l'Ecole Polytechnique (1975); Neveu, J.: Evolution Markoviennes. Séminaire sur les processus markoviens à une infinité de particules, Ecole Polytechnique (1974);

Spitzer, F.: Random fields and interacting particle systems MAA Seminar. Williamstown, Massachusetts (1971)

9. Feller, W.: An introduction to probability theory and its applications. I, II, 3rd edition. New York: Wiley and Sons 1967 ;

Simon, B.: Functional integration and quantum physics. London, New York: Academic Press 1979

10. Billingsley, P.: Convergence of probability measures. New York: Wiley and Sons 1968

11. Metivier, M. : Sufficient conditions for tightness and weak convergence of a sequence of processes. Internal report, University of Minnesota, Minneapolis;

Rebolledo, R.: La méthode des martingales appliquées à la convergence en loi des processus. Mém. Soc. Math. Fr. (to appear)

12. Spitzer, F.: Trans. Am. Math. Soc. 198, 191-199 (1974)

13. Davies, E.B.: J. Stat. Phys. 18, 161-170 (1978)

14. Kingman, J.F.C.: J. Appl. Prob. 6, 1-18 (1969)

Communicated by J. Lebowitz

Received May 5, 1980, in revised form December 16, 1980 
